\title{
Prothrombotic genotypes and risk of major bleeding in patients with incident venous thromboembolism
}

Running title: Prothrombotic genotypes and major bleeding risk

Håkon S. Johnsen ${ }^{1}$, Esben Bjøri ${ }^{1}$, Kristian Hindberg ${ }^{1}$, Sigrid K. Brækkan ${ }^{1,2}$, Vânia M. Morelli ${ }^{1}$, John-Bjarne Hansen ${ }^{1,2}$

${ }^{1}$ K.G Jebsen - Thrombosis Research and Expertise Center (TREC), Department of Clinical Medicine, UiT The Arctic University of Norway, ${ }^{2}$ Division of Internal Medicine, University Hospital of North Norway, Troms $\varnothing$, Norway.

Correspondence to: Håkon Sandbukt Johnsen M.D, K.G. Jebsen - Thrombosis Research and Expertise Center (TREC), Department of Clinical Medicine, UiT - The Arctic University of Norway, N-9037, Norway.

E-mail: hakon.s.johnsen@uit.no

Telephone: +4799247706

Abstract word count: 249

Text word count: 4096 (introduction, methods, results and discussion)

Number of tables and figures: 7 (5 tables and 2 figures)

Number of supplemental data: 1 supplementary table

Number of references: 31 


\section{Highlights}

- Whether multiple prothrombotic genotypes reduce bleeding risk during anticoagulation in patients with venous thromboembolism (VTE) is unknown

- The five genotypes most strongly associated with VTE risk were assessed in 676 incident VTE cases

- An increasing number of prothrombotic risk alleles was not associated with major bleeding risk

- Prothrombotic genotypes may not help to stratify major bleeding risk during anticoagulation after a VTE 


\begin{abstract}
Background: Genotypes associated with venous thromboembolism (VTE) may protect against bleeding due to a hypercoagulable state. Whether the risk of major bleeding is reduced in parallel with an increasing number of prothrombotic genotypes during anticoagulant treatment in VTE remains unknown.
\end{abstract}

Objectives: To investigate the association between multiple prothrombotic genotypes and risk of major bleeding in patients with VTE.

Methods: Patients with incident VTE ( $\mathrm{n}=676)$ derived from the Troms $\varnothing$ Study were genotyped for rs6025 (F5), rs1799963 (F2), rs8176719 (ABO), rs2066865 (FGG) and rs2036914 (F11) single nucleotide polymorphisms (SNPs). Major bleeding events were recorded during the first year after VTE according to the International Society on Thrombosis and Haemostasis criteria. Cox-regression was used to calculate hazard ratios with $95 \%$ confidence intervals (CIs) for major bleeding adjusted for age, sex and duration of anticoagulation according to individual prothrombotic SNPs and categories of risk alleles (5-SNP score; 0-1, 2, 3 and $\geq 4$ ). Results: In total, 50 patients experienced major bleeding (incidence rate: 9.5/100 person-years, 95\% CI 7.2-12.5). The individual SNPs and number of risk alleles were not associated with major bleeding risk. The hazard ratios for major bleeding per category increase of genetic risk score were 1.0 (95\% CI 0.8-1.3) for the total study population and 1.1 (95\% CI 0.8-1.5) when patients with active cancer were excluded. Analyses restricted to the first 3 months after VTE yielded similar results.

Conclusion: Our findings suggest that an increasing number of prothrombotic risk alleles is not protective against major bleeding in VTE patients during anticoagulation.

Keywords: venous thromboembolism, anticoagulation, genotype, hemorrhage, bleeding 


\section{Introduction}

Bleeding is a dominant and potentially serious complication in patients undergoing anticoagulant treatment for venous thromboembolism (VTE). ${ }^{1}$ The annual rates of major bleeding (MB) in VTE patients during anticoagulant treatment have been reported to vary from $1 \%$ to approximately $10 \%$ depending on the type of anticoagulant, patient characteristics and study setting (clinical trials versus daily care). ${ }^{2-7}$ An accurate risk prediction of MB is crucial to make informed decisions on VTE management and particularly to guide the treatment duration in unprovoked VTE towards the highest net benefit. However, the current prediction models for MB in VTE are mainly based on clinical factors, and do not discriminate well between VTE patients at high and low risk of MB in validation studies. ${ }^{8-11}$ Extended knowledge on risk factors associated with MB in VTE patients is a key step to improve risk stratification of MB events during anticoagulant treatment.

Single nucleotide polymorphisms (SNPs) associated with increased risk of VTE, such as factor V Leiden (FVL), prothrombin G20210A, and non-O blood type, ${ }^{12}$ could be plausible candidate risk factors for MB. The prothrombotic genotypes have been shown to be associated with a hypercoagulable state ${ }^{12}$ and could therefore have the potential to counteract the risk of bleeding related to anticoagulation in VTE patients. For instance, FVL is associated with resistance to the natural anticoagulant protein $C,{ }^{13}$ prothrombin $\mathrm{G} 20210 \mathrm{~A}$ with increased plasma levels of prothrombin, ${ }^{14}$ and non-O blood type with increased plasma levels of von Willebrand factor and coagulation factor VIII. ${ }^{15}$ The few studies addressing the impact of prothrombotic genotypes on the risk of $\mathrm{MB}$ during anticoagulant therapy have suggested a decreased risk of $\mathrm{MB}$ in carriers of $\mathrm{FVL}^{16}$ and non-O blood types. ${ }^{17}$

In the context of VTE, de Haan and colleagues developed a genetic risk score originally based on 31 VTE-associated SNPs that was shown to improve the risk prediction of first lifetime 
VTE. ${ }^{18}$ The number of prothrombotic risk alleles included in the genetic score was dosedependently associated with VTE risk (i.e. the more risk alleles present, the higher the risk of VTE). A similar predictive performance and dose-response relationship was found when the authors conceived a more parsimonious score consisting of the 5 most strongly VTE-associated SNPs, i.e. rs6025 (FVL) in F5, rs1799963 (prothrombin G20210A) in F2, rs8176719 (non-O blood type) in $A B O$, rs2066865 in the fibrinogen gamma gene $(F G G)$ and rs2036914 in $F 11 .^{18}$ Given that the mechanisms by which multiple prothrombotic genotypes affect the thrombosis risk likely reflect different pathways in blood coagulation, we hypothesized that an increasing number of prothrombotic risk alleles would have a dose-dependent protective effect on the risk of MB. To the best of our knowledge, no previous study has assessed the impact of multiple prothrombotic genotypes on the risk of MB. Therefore, we aimed to investigate the association between multiple prothrombotic genotypes and risk of $\mathrm{MB}$ during the first year after an incident VTE.

\section{Methods}

\section{Study population}

The study population originated from the Troms $\varnothing$ Study, a single-center, population-based prospective cohort, with repeated health surveys of the inhabitants of Troms $\varnothing$, Norway. ${ }^{19}$ Study participants were recruited from the fourth (1994-1995), fifth (2001-2002) and sixth (2007-2008) surveys of the Troms $\emptyset$ Study. The overall attendance rates were high, achieving $77 \%$ in the fourth survey, $79 \%$ in the fifth survey, and $66 \%$ in the sixth survey. In total, there were 30371 unique individuals aged 25-97 years who participated in at least one of the surveys. The study was approved by the Regional Committee of Research and Medical Health Ethics, and all study participants provided informed written consent. 
From the date of inclusion in one of the three surveys until the end of follow-up at December 31, 2012, all potential first lifetime venous thromboembolism (VTE) events were identified by searching the hospital discharge diagnosis registry, the autopsy registry, and the radiology procedure registry at the University hospital of North Norway (UNN). Identified cases were adjudicated by trained personnel and deemed as validated when signs and symptoms of deep vein thrombosis (DVT) or pulmonary embolism (PE) were combined with objective confirmation by radiological procedures, and resulted in a VTE diagnosis requiring treatment, as described in detail previously. ${ }^{20}$ A total of 737 objectively confirmed VTE cases were identified during follow-up. Of these, 45 did not have blood samples available or of sufficient quality for DNA analysis, and 16 were not successfully genotyped for one or more of the SNPs of interest. Therefore, 676 VTE cases were included in our study.

\section{Clinical characteristics of VTE events}

Information on clinical and provoking factors at the time of and 12 weeks preceding the VTE diagnosis was obtained for all eligible patients by review of medical records at the UNN. In the case of a concurrent DVT and PE diagnosis, the VTE event was classified as a PE. Each VTE event was further classified as provoked if one or more of the following risk factors were present: major surgery, trauma or acute medical conditions (acute myocardial infarction, ischemic stroke, or major infectious disease) within 12 weeks prior to VTE event, marked immobilization (bed confinement $>3$ days, wheel-chair, or long distance travel exceeding 4 hours within the last 14 days prior to VTE event), or any other factor specifically described in the medical records to have provoked the VTE (e.g. intravascular catheter). Presence of known active cancer at the time of VTE diagnosis was regarded as a provoked VTE. 
To account for type and duration of VTE treatment, we considered the planned treatment (i.e. heparin or vitamin $\mathrm{k}$ antagonist [VKA]) and duration of anticoagulation that were stated by the attending physicians in the medical records at the time of VTE diagnosis. Duration of anticoagulant therapy was categorized into $3,6,12$, and more than 12 months, as previously described. $^{21}$

Assessment of major bleeding (MB) events

The medical records for all participants were searched for MB events occurring during the 365 days following the VTE. All second-line care and advanced emergency medicine, such as transfusion of blood products, is exclusively provided by the UNN. The UNN is situated in the middle of Troms $\varnothing$ municipality, with a vicinity of approximately 250 kilometers to the nearest hospital providing comparable health-care functions. Two reviewers (trained medical personnel from the UNN) adjudicated the bleeding events independently in accordance with the criteria proposed by the International Society on Thrombosis and Hemostasis (ISTH). ${ }^{22}$ In short, a bleeding event that was fatal, and/or symptomatic in a critical area or organ, and/or causing a fall in hemoglobin level of $\geq 20 \mathrm{~g} / \mathrm{L}$, or requiring transfusion of $\geq 2$ units of whole blood or red blood cells, was considered major. In case of disagreement, the event was discussed in an endpoint committee (HSJ and JBH) to reach consensus.

\section{Genotyping and quality control}

Blood samples were collected from an antecubital vein at enrollment in the Troms $\varnothing$ Study and the initial preparation of samples was done at the department of Clinical Chemistry at the UNN. DNA was isolated from whole blood and stored at $-70^{\circ} \mathrm{C}$ at the national CONOR biobank, located at the HUNT Biobank in Levanger, Norway. As previously described, ${ }^{23}$ we genotyped 
rs6025 (FVL) in $F 5$, rs 1799963 (prothrombin G20210A) in $F 2$, rs8176719 (non-O blood type) in $A B O$ and rs2036914 in F11 with the Sequenom platform, and rs2066865 in $F G G$ with the TaqMan platform. For Sequenom, which uses single-base extension followed by mass spectrometry to measure the molecular mass of the extended primer, samples were genotyped with the Sequenom iPlex Gold Assay according to the recommended protocol, with an initial input of 10-20 ng of DNA, and were analyzed with the MassARRAY Analyzer 4. Only genotypes with a high quality score of "A - Conservative" or "B - Moderate" were used. When multiple attempts were made to genotype an individual, one of the highest-quality genotypes across all attempts was chosen for each SNP. For TaqMan, an initial input of $100 \mathrm{ng}$ of DNA was used. Samples were genotyped with the Applied Biosystems 7900HT (Foster City, CA, USA) according to the recommended protocol, and processed with SDS 2.4 (Thermo Fisher, Foster City, CA, USA).

Subjects were considered carriers of the prothrombotic risk gene when one or two risk alleles were present. Individual SNP assessment was done without differentiation between heterozygous and homozygous carriers due to small number of homozygotes for most SNPs. According to literature, risk alleles were defined as the variant associated with increased VTE risk. ${ }^{12,18}$ Among the SNPs studied, rs2036914 in F11 was the only one with a minor allele associated with reduced VTE risk, and in this case, we considered the common allele as the risk allele. Similar to de Haan et al., a 5- SNP score was conceived by counting the number of risk alleles from the five sequenced SNPs, with a theoretical maximum number of ten risk alleles for an individual (i.e. two risk alleles were counted for homozygous carriers). ${ }^{18}$

\section{Statistical analysis}


Subjects were followed from the date of their first lifetime VTE to the date of an incident MB, death, migration, or end of follow-up (i.e. 365 days after the first VTE), whichever came first. Subjects who died or migrated out of the municipality of Troms $\varnothing$ were censored at the date of the respective event. Statistical analyses were performed using STATA version 15.0 (Stata Corporation LP, College Station, TX, USA).

Based on the 5-SNP score, we created categories of $0-1,2,3$ and $\geq 4$ risk alleles instead of performing analyses per-risk-allele, due to small numbers. Crude incidence rates (IRs) with 95\% confidence intervals (CIs) of MB were calculated for the individual SNPs and categories of the 5SNP score, and expressed as number of events per 100 person-years at risk. Cox proportional hazards regression models were used to calculate hazard ratios (HRs) with 95\% CIs for MB according to the individual SNPs (reference: 0 risk allele) and categories of the 5-SNP score (reference: 0-1 risk allele). HRs were adjusted for age and sex in a first model with the addition of planned duration of anticoagulant therapy in a second model. Risk estimates were adjusted for treatment duration because knowledge of thrombophilia or family history of early VTE onset might have resulted in the admitting physician planning longer treatment with anticoagulation, which could affect the risk of MB. In a separate sensitivity analysis, we also calculated HRs for MB by risk alleles restricted to the first three months after VTE diagnosis, when all subjects would be on anticoagulant treatment. For further sensitivity purposes, we assessed the risk of MB after excluding subjects with known cancer at time of VTE diagnosis, as cancer patients might be at increased risk of bleeding during anticoagulation. ${ }^{24}$ Finally, we performed subgroup analyses according to clinical presentation (i.e. DVT or PE) and presence of provoking risk factors (i.e. provoked or unprovoked VTE events). The proportional hazard assumption was assessed by evaluating the parallelism of the log-log survivor function by categories of number of risk alleles, and tested with Schoenfeld residuals. 
The 1-year cumulative incidences of MB across categories of the 5-SNP score were calculated and visualized in one minus Kaplan-Meier (1-KM) plots for the overall population and after excluding those with cancer at the time of VTE diagnosis.

\section{Results}

Baseline characteristics according to categories of prothrombotic risk alleles derived from the 5SNP score are shown in Table 1. In the study population, the mean age and body mass index, the proportion of DVT and PE, and the planned duration of anticoagulation did not substantially differ across categories of risk alleles. Among patients in the highest category of the genetic score (i.e. $\geq 4$ risk alleles), the proportion of men, subjects with active cancer at the time of VTE diagnosis and those treated with heparin tended to be lower, and the VTE events were more likely to be unprovoked in comparison with patients in the other categories.

Among the 676 patients with incident VTE, 50 had an MB within 1 year after the VTE event, resulting in an overall IR of 9.5 per 100 person-years (95\% CI 7.2-12.5). The median time from incident VTE to MB was 33 days (interquartile range 11-180 days). Characteristics of MB events are presented in Table 2, showing that nearly half of the MB episodes was classified as major due to symptomatic bleeding in a critical area or organ.

As depicted in Fig. 1, the distribution of VTE patients across number of prothrombotic risk alleles of the 5-SNP score ranged from 0 to 6 , with a median number of 3 . The IRs and HRs for MB according to individual SNPs and categories of risk alleles of the 5-SNP score in the overall population are shown in Table 3. None of the individual SNPs were associated with risk of MB. In the age- and sex-adjusted models, HRs for MB were within the range 1.0 to 1.1 for rs6025 (F5), rs8176719 (ABO), and rs2066865 (FGG). Further adjustment for planned duration of anticoagulation did not materially change risk estimates. For rs 2036914 in F11, the HR for 
MB was 2.0 (95\% CI 0.8-5.0) in the fully adjusted model, and for rs 1799963 in $F 2$, no individual carrying $\geq 1$ risk allele experienced MB. Similarly to the individual SNPs, the number of prothrombotic risk alleles in the 5-SNP score had no impact on the risk of MB (Table 3). In the model adjusted for age, sex, and planned duration of anticoagulation, the HR for MB per category increase of genetic risk score was 1.0 (95\% CI 0.8-1.3). Compared to subjects with 0-1 risk allele, the HRs for MB were 1.7 (95\% CI 0.7-4.5), 1.6 (95\% CI 0.6-4.0) and 1.3 (95\% CI 0.53.5 ) in carriers of 2,3 and $\geq 4$ risk alleles, respectively. When analyses were restricted to the first 3 months of follow-up, again there was no consistent association of the individual SNPs or categories of the 5-SNP score with risk of MB (Supplementary Table 1).

In sensitivity analysis, in which we excluded patients with known active cancer at the time of VTE diagnosis, results were essentially similar to the main analysis, with no significant associations between prothrombotic genotypes and risk of MB (Table 4). Table 5 shows the risk of MB by categories of the 5-SNP score according to subgroups of VTE (provoked, unprovoked, DVT and PE). For unprovoked VTE, the HR for MB per category increase of genetic risk score was 1.0 (95\% CI 0.6-1.6) in the fully adjusted model. Analyses of the other subgroups did not reveal any consistent association of $\mathrm{MB}$ risk across number of prothrombotic risk alleles.

The 1-year cumulative incidences of MB across categories of the 5-SNP score were estimated by 1-KM for the overall study population (Fig. 2A) and after excluding subjects with active cancer at the time of VTE diagnosis (Fig. 2B). As shown in Fig. 2A, the majority of the MB events occurred in the first 3 months after the VTE. The 3-month cumulative incidences of MB for subjects with $0-1,2,3$ and $\geq 4$ risk alleles were $2.0 \%$ (95\% CI 0.5-7.9), $6.6 \%$ (95\% CI 3.7-11.5), 6.7\%, (95\% CI 3.9-11.2), and 4.1\% (95\% CI 2.0-8.5), respectively. Similar results were found in patients without cancer (Fig. 2B). 


\section{Discussion}

In this population-based cohort of 676 patients followed for 1 year after their first lifetime VTE, we investigated whether the risk of $\mathrm{MB}$ was stepwise reduced in parallel with an increasing number of prothrombotic risk alleles. Using a genetic risk score based on five prothrombotic SNPs, we found that the number of risk alleles was not associated with risk of MB. Likewise, none of the individual prothrombotic SNPs were associated with risk of MB. Exclusion of patients with active cancer at the time of VTE diagnosis, restriction to the first 3 months after VTE diagnosis, or subgroup analyses stratified by unprovoked or provoked VTE and location (i.e. DVT or PE) yielded essentially similar results. Our findings suggest that a hypercoagulable state driven by prothrombotic genotypes is not able to restrain a severe bleeding associated with anticoagulant treatment.

In our study, originated from the general population, the overall rate of MB was 9.5 per 100 person-years, which was considerably higher than the rate of about 1.0 per 100 person-years reported in randomized controlled trials (RCTs) comprising VTE patients. ${ }^{2}$ However, our results are not unexpected, as unselected patients derived from the general population have more often serious comorbidities and are managed under less intensive surveillance as compared to patients selected into RCTs. Moreover, RCTs on the efficacy and safety of anticoagulant treatment tend to exclude patients with a bleeding predisposition. Of note, $24 \%$ of our patients (159 out of 676) had active cancer at the time of VTE diagnosis, which is a well-known risk factor for bleeding during anticoagulation. ${ }^{24}$ Our rate of $\mathrm{MB}$ is in line with a similar prospective cohort consisting of 842 VTE patients treated with anticoagulant therapy during daily care, of whom $21.5 \%$ had known cancer. ${ }^{7}$ In the above mentioned study, the rate of MB was 10.6 per 100 person-years, and it was especially high in analysis restricted to VTE patients with cancer (15.7 per 100 person-year). Another noteworthy finding of our study was the fact that the majority of the MB events occurred 
within the first 3 months after VTE. These results are in accordance with previous data ${ }^{25}$ and with the notion that patients with an underlying predisposition to bleeding are more likely to develop a MB event early after initiation of anticoagulation. Still, the prothrombotic genotypes studied were not associated with risk of MB even within the initial 3 months after VTE when the majority of MB events occurred.

The association between prothrombotic genotypes and risk of MB has been scarcely investigated in a VTE population under anticoagulant treatment. ${ }^{16,17}$ In a Dutch case-control study that used data from the FACTors in ORal anticoagulation Safety (FACTORS) study, ${ }^{26}$ Garcia et al. ${ }^{17}$ found that the risk of MB during anticoagulation with VKAs in non-OO blood group carriers was $30 \%$ lower than in carriers of OO blood group. Even though risk estimates pointed towards a protective effect (odds ratios $=0.7,95 \%$ CI $0.4-1.1$ ), the $95 \%$ CIs were somehow wide and included unity in the aforementioned report. ${ }^{17}$ Furthermore, only $20 \%$ of 110 cases with MB and 220 controls without MB presented VTE as an indication for anticoagulation, and bleeding leading to hospitalization was also among the criteria to define MB. ${ }^{17,26}$ Admission to hospital can be influenced by other important factors, such as support in the community and presence of comorbidities, ${ }^{22}$ thus not necessarily reflecting the severity of a bleeding event. In another study, Franchini et al. assessed the ABO blood group phenotypes instead of genotypes in 183 cases with bleeding complications and 366 controls without bleeding during treatment with VKA, of whom about $25 \%$ had VTE. ${ }^{27}$ Even though bleeders met at most grade 2 (mild blood loss) of a 4-grade World Health Organization grading system, no association between ABO blood group and bleeding was found. ${ }^{27}$ Our results interpreted in light of the existing studies indicate that there are no consistent data supporting a protective effect of non-O blood type on the risk of MB in VTE patients during anticoagulation. 
In a study derived from the RIETE-registry comprising 10139 VTE patients tested for thrombophilia, Tzoran et al. ${ }^{16}$ investigated the impact of FVL on the risk of MB during anticoagulation. Compared to noncarriers, FVL carriers $(n=1384)$ had a 50\% $(\mathrm{HR} 0.50,95 \%$ 0.25-0.99) lower risk of MB. It is important to address that patients undergoing thrombophilia testing generally do not share the clinical characteristics of unselected VTE patients derived from a general population. For instance, in the above mentioned study, ${ }^{16}$ the proportion of VTE patients with cancer was relatively low (6\%-11\% among carriers and noncarriers of the prothrombotic genotypes studied) as compared to the present study (24\%). Therefore, the study from Tzoran et al. is not necessarily comparable to our population-based cohort.

It is of interest that minor bleeding has been shown to be associated with subsequent increased risk of $\mathrm{MB}$ in patients treated with VKA, independent of the quality of anticoagulation and other known risk factors. ${ }^{28}$ The association was confirmed and further explored by van Rein et al. ${ }^{29}$ who used a case-crossover design to untangle the nature of such an association. Results from the case-crossover study suggested that minor bleeds could be markers for fixed risk factors for MB events, like genetic variants affecting blood coagulation. For instance, the mild hypercoagulable state associated with $\mathrm{FVL}^{13}$ has been proposed to have offered evolutionary advantages in face of life-threatening bleeding, such as during childbirth, warfare, or other activities carrying high risks of trauma. ${ }^{30}$ However, in the context of bleeding related to anticoagulant treatment after a VTE, our study revealed that neither the individual SNPs nor the increasing number of prothrombotic risk alleles included in the 5-SNP score ${ }^{18}$ were protective against an MB event in VTE patients. We can speculate that the mild hypercoagulability driven by FVL and the other prothrombotic genotypes would probably not be able to balance the mechanisms that induce a MB associated with anticoagulant treatment. Alternatively, our findings could be explained by a phenomenon called index event bias. ${ }^{31}$ VTE is a result of 
multiple risk factors, and a selection of only incident VTE events (as in a cohort of VTE patients) may induce dependence between these risk factors. For example, an inverse association between carriership of prothrombotic genotypes and other prothrombotic factors could arise in the incident VTE population. If these other prothrombotic factors are also protective against bleeding, the effect measure of the risk of MB in carriers of prothrombotic genotypes compared to noncarriers may be shifted towards the null. Taken together, based on our findings, it is unlikely that the prothrombotic genotypes included in the 5-SNP score would be of clinical relevance to improve discrimination between those with high and low risk of MB during anticoagulant treatment in VTE patients.

The inclusion of subjects derived from the general population is among the main strengths of the present study. In comparison to subjects included in RCTs, the clinical characteristics, comorbidities and prevalence of cancer in our study participants are similar to those found in real-life patients, thereby increasing the external validity of our results. Other strengths include the complete and validated registry of VTE events, the exclusivity of UNN as the sole health care provider, likely to receive all relevant MB events, and the strict criteria used to define MB based on the ISTH recommendations. ${ }^{22}$ Limitations also need to be addressed. Given our sample size and number of MB cases, the statistical power could have been limited to detect slight protective effects of prothrombotic genotypes, especially among those in the uppermost category of the genetic score (i.e. carriers of $\geq 4$ risk alleles) and in subgroups of VTE. Of note, the risk estimates of MB were above 1.0 in some subgroups, but the $95 \%$ CIs of these estimates were considerably wide and included unity. It is important to address, that when the 5-SNP score was analyzed per category increase in genetic risk score, the risk estimates were 1.0 or around 1.0 both in overall and subgroup analyses, as well as in sensitivity analyses, thereby suggesting no protective effect of prothrombotic genotypes on MB risk in VTE patients. We were not able to perform analyses 
stratified by planned type of anticoagulant treatment (i.e. heparin or heparin and VKA) due to the low number of participants in the heparin group $(\mathrm{n}=98)$ and the low number of MB events $(\mathrm{n}=$ 10). Moreover, we did not have information on concomitant use of drugs that might have affected the bleeding risk, such as antiplatelet agents. However, it is very unlikely that the type of anticoagulant, and the use of concomitant drugs, would differ across categories of prothrombotic risk alleles and thereby serve as potential confounders for the relationship between prothrombotic genotypes and risk of MB. In this study, we did not have access to information on the actual duration of anticoagulant treatment. Nonetheless, when we restricted our analyses to the first three months after VTE diagnosis (i.e. a period during which all patients would be on anticoagulant treatment), results were essentially similar to the overall analyses, showing no consistent association between prothrombotic risk alleles and risk of MB. Finally, an increasing number of prothrombotic risk alleles could still be protective against clinically relevant nonmajor bleedings (CRNMB). However, our study was not designed to evaluate CRNMB, and future research is required to address this question.

In conclusion, our study suggests that prothrombotic genotypes, evaluated either as individual SNPs or as number of risk alleles included in a 5-SNP score, do not protect against MB in VTE patients during anticoagulant treatment. Our findings further suggest that assessment of prothrombotic genotypes is not helpful to improve patient stratification of MB risk during anticoagulation for a VTE. 


\section{Addendum}

H.S. Johnsen contributed to data collection, statistical analyses, data interpretation, and drafted the manuscript.

E. Bjøri and K. Hindberg contributed to statistical analyses, data interpretation, and revision of the manuscript.

S.K. Brækkan contributed to data collection, data interpretation and critical revision of the manuscript.

V.M. Morelli contributed to data interpretation and critical revision of the manuscript.

J.B. Hansen provided study concept and design, and contributed to data interpretation and critical revision of the manuscript.

\section{Acknowledgements}

The K. G. Jebsen Thrombosis Research and Expertise Center is supported by an independent grant from Stiftelsen Kristian Gerhard Jebsen.

H. S. Johnsen receives a grant from the North Norwegian regional health authorities (HelseNord).

\section{Disclosure of Conflict of Interests}

The authors declare that they have no conflicts of interest. 


\section{References}

1. Schulman S, Beyth RJ, Kearon C, Levine MN, American College of Chest P. Hemorrhagic complications of anticoagulant and thrombolytic treatment: American College of Chest Physicians Evidence-Based Clinical Practice Guidelines (8th Edition). Chest. 2008;133(6 Suppl):257S-298S.

2. Ost D, Tepper J, Mihara H, Lander O, Heinzer R, Fein A. Duration of anticoagulation following venous thromboembolism: a meta-analysis. JAMA. 2005;294(6):706-715.

3. Veeger NJ, Piersma-Wichers M, Tijssen JG, Hillege HL, van der Meer J. Individual time within target range in patients treated with vitamin $\mathrm{K}$ antagonists: main determinant of quality of anticoagulation and predictor of clinical outcome. A retrospective study of 2300 consecutive patients with venous thromboembolism. Br J Haematol. 2005;128(4):513-519.

4. Beyer-Westendorf J, Forster K, Pannach S, et al. Rates, management, and outcome of rivaroxaban bleeding in daily care: results from the Dresden NOAC registry. Blood. 2014;124(6):955-962.

5. Khan F, Datta YH. Risk of bleeding during long-term anticoagulation with warfarin: a tertiary care center experience. Blood Coagul Fibrinolysis. 2015;26(1):110-112.

6. Lutsey PL, Zakai NA, MacLehose RF, et al. Risk of hospitalised bleeding in comparisons of oral anticoagulant options for the primary treatment of venous thromboembolism. British journal of haematology. 2019;185(5):903-911. 
7. Prandoni $\mathrm{P}$, Lensing AW, Piccioli $\mathrm{A}$, et al. Recurrent venous thromboembolism and bleeding complications during anticoagulant treatment in patients with cancer and venous thrombosis. Blood. 2002;100(10):3484-3488.

8. Piovella C, Dalla Valle F, Trujillo-Santos J, et al. Comparison of four scores to predict major bleeding in patients receiving anticoagulation for venous thromboembolism: findings from the RIETE registry. Intern Emerg Med. 2014;9(8):847-852.

9. Riva N, Bellesini M, Di Minno MN, et al. Poor predictive value of contemporary bleeding risk scores during long-term treatment of venous thromboembolism. A multicentre retrospective cohort study. Thrombosis and haemostasis. 2014;112(3):511-521.

10. Klok FA, Niemann C, Dellas C, Hasenfuss G, Konstantinides S, Lankeit M. Performance of five different bleeding-prediction scores in patients with acute pulmonary embolism. Journal of thrombosis and thrombolysis. 2016;41(2):312-320.

11. Palareti G, Antonucci E, Mastroiacovo D, et al. The American College of Chest Physician score to assess the risk of bleeding during anticoagulation in patients with venous thromboembolism. Journal of thrombosis and haemostasis : JTH. 2018;16(10):19942002.

12. Morange PE, Tregouet DA. Current knowledge on the genetics of incident venous thrombosis. J Thromb Haemost. 2013;11 Suppl 1:111-121.

13. Bertina RM, Koeleman BP, Koster $\mathrm{T}$, et al. Mutation in blood coagulation factor $\mathrm{V}$ associated with resistance to activated protein C. Nature. 1994;369(6475):64-67.

14. Poort SR, Rosendaal FR, Reitsma PH, Bertina RM. A common genetic variation in the 3'untranslated region of the prothrombin gene is associated with elevated plasma 
prothrombin levels and an increase in venous thrombosis. Blood. 1996;88(10):36983703.

15. Morelli VM, de Visser MC, van Tilburg NH, et al. ABO blood group genotypes, plasma von Willebrand factor levels and loading of von Willebrand factor with A and B antigens. Thrombosis and haemostasis. 2007;97(4):534-541.

16. Tzoran I, Papadakis M, Brenner B, et al. Outcome of Patients with Venous Thromboembolism and Factor V Leiden or Prothrombin 20210 Carrier Mutations During the Course of Anticoagulation. The American journal of medicine. 2017;130(4):482.e481482.e489.

17. Garcia AA, van der Heijden JF, Meijers JC, Rosendaal FR, Reitsma PH. The relationship between $\mathrm{ABO}$ blood group and the risk of bleeding during vitamin $\mathrm{K}$ antagonist treatment. J Thromb Haemost. 2006;4(6):1418-1420.

18. de Haan HG, Bezemer ID, Doggen CJ, et al. Multiple SNP testing improves risk prediction of first venous thrombosis. Blood. 2012;120(3):656-663.

19. Jacobsen BK, Eggen AE, Mathiesen EB, Wilsgaard T, Njolstad I. Cohort profile: the Tromso Study. International journal of epidemiology. 2012;41(4):961-967.

20. Braekkan SK, Borch KH, Mathiesen EB, Njolstad I, Wilsgaard T, Hansen JB. Body height and risk of venous thromboembolism: The Tromso Study. Am J Epidemiol. 2010;171(10):1109-1115.

21. Johnsen HS, Hindberg K, Bjori E, et al. D-Dimer Measured at Diagnosis of Venous Thromboembolism is Associated with Risk of Major Bleeding. TH Open. 2019;3(1):e77e84. 
22. Schulman S, Kearon C, Subcommittee on Control of Anticoagulation of the S, Standardization Committee of the International Society on T, Haemostasis. Definition of major bleeding in clinical investigations of antihemostatic medicinal products in nonsurgical patients. J Thromb Haemost. 2005;3(4):692-694.

23. Horvei LD, Braekkan SK, Smith EN, et al. Joint effects of prothrombotic genotypes and body height on the risk of venous thromboembolism: the Tromso study. J Thromb Haemost. 2018;16(1):83-89.

24. Kearon C, AkI EA, Ornelas J, et al. Antithrombotic Therapy for VTE Disease: CHEST Guideline and Expert Panel Report. Chest. 2016;149(2):315-352.

25. Linkins LA, Choi PT, Douketis JD. Clinical impact of bleeding in patients taking oral anticoagulant therapy for venous thromboembolism: a meta-analysis. Ann Intern Med. 2003;139(11):893-900.

26. van der Heijden JF, Rekke B, Hutten BA, et al. Non-fatal major bleeding during treatment with vitamin $\mathrm{K}$ antagonists: influence of soluble thrombomodulin and mutations in the propeptide of coagulation factor IX. J Thromb Haemost. 2004;2(7):1104-1109.

27. Franchini M, Crestani S, Frattini F, Mengoli C, Bonfanti C. Relationship between ABO blood group and bleeding complications in orally anticoagulated patients. J Thromb Haemost. 2012;10(8):1688-1691.

28. Veeger NJ, Piersma-Wichers M, Meijer K, Hillege HL. Minor bleeds alert for subsequent major bleeding in patients using vitamin K antagonists. Br J Haematol. 2011;153(4):508514. 
29. van Rein N, le Cessie S, van Vliet IP, et al. Increased risk of major bleeding after a minor bleed during treatment with vitamin $\mathrm{K}$ antagonists is determined by fixed common risk factors. J Thromb Haemost. 2016;14(5):948-952.

30. Zivelin A, Griffin JH, Xu X, et al. A single genetic origin for a common Caucasian risk factor for venous thrombosis. Blood. 1997;89(2):397-402.

31. Dahabreh IJ, Kent DM. Index event bias as an explanation for the paradoxes of recurrence risk research. JAMA. 2011;305(8):822-823. 


\section{Tables and figures}

Table 1 Baseline characteristics of venous thromboembolism (VTE) cases across categories of the 5-single nucleotide polymorphism (SNP) score

\begin{tabular}{|c|c|c|c|c|}
\hline & \multicolumn{4}{|c|}{ Number of risk alleles } \\
\hline & $\begin{array}{l}0-1 \\
(\mathrm{n}=109)\end{array}$ & $\begin{array}{l}2 \\
(\mathrm{n}=179)\end{array}$ & $\begin{array}{l}3 \\
(\mathrm{n}=205)\end{array}$ & $\begin{array}{l}\geq 4 \\
(\mathrm{n}=183)\end{array}$ \\
\hline Age (years), mean $\pm \mathrm{SD}$ & $71 \pm 12$ & $68 \pm 14$ & $69 \pm 14$ & $67 \pm 13$ \\
\hline Sex (males) & $46.0(50)$ & $51.0(92)$ & $46.0(94)$ & $40.0(74)$ \\
\hline $\mathrm{BMI}\left(\mathrm{kg} / \mathrm{m}^{2}\right)^{\mathrm{a}}$, mean $\pm \mathrm{SD}$ & $27.2 \pm 4.7$ & $27.1 \pm 4.8$ & $27.4 \pm 4.6$ & $27.3 \pm 4.3$ \\
\hline DVT & $56(61)$ & $56(101)$ & $59(121)$ & $57(104)$ \\
\hline $\mathrm{PE} \pm \mathrm{DVT}$ & $44(48)$ & $44(78)$ & $41(84)$ & $43(79)$ \\
\hline Provoked VTE & $63(69)$ & $63(112)$ & 57 (117) & $51(94)$ \\
\hline Unprovoked VTE & $37(40)$ & $37(67)$ & $43(88)$ & $49(89)$ \\
\hline Active cancer $^{\mathrm{b}}$ & $27(29)$ & $28(50)$ & $25(52)$ & $15(28)$ \\
\hline Initial thrombolytic therapy & $6(7)$ & $4(8)$ & $5(10)$ & $5(9)$ \\
\hline \multicolumn{5}{|l|}{ Planned treatment type } \\
\hline Heparin $^{c}$ & $16(17)$ & $18(32)$ & $15(30)$ & $10(19)$ \\
\hline Heparin and VKA ${ }^{\mathrm{d}}$ & $68(74)$ & $69(124)$ & $75(153)$ & $80(147)$ \\
\hline \multicolumn{5}{|l|}{$\begin{array}{l}\text { Planned duration of } \\
\text { anticoagulation }\end{array}$} \\
\hline$\leq 3$ months & $36(39)$ & $36(65)$ & $36(73)$ & $37(67)$ \\
\hline > 3 including 6 months & $32(35)$ & $33(59)$ & $37(76)$ & $33(61)$ \\
\hline$>6$ including 12 months & $22(24)$ & $26(47)$ & $20(42)$ & $19(35)$ \\
\hline$>12$ months & $10(11)$ & $5(8)$ & $7(14)$ & $11(20)$ \\
\hline
\end{tabular}

Abbreviations: Body mass index (BMI); DVT, deep vein thrombosis; PE, pulmonary embolism;

SD, standard deviation; VKA, vitamin K antagonist

Categorical variables are shown as percentages with numbers in brackets, \% (n)

${ }^{a} \mathrm{BMI}$ was calculated as weight in kilograms divided by the square of height in meters $\left(\mathrm{kg} / \mathrm{m}^{2}\right)$

${ }^{\mathrm{b}}$ Active cancer at the time of VTE diagnosis

${ }^{c}$ Low molecular weight or unfractionated heparin

${ }^{\mathrm{d}}$ Low molecular weight or unfractionated heparin with VKA treatment 
Table 2 Characteristics of major bleeding in patients with venous thromboembolism

\begin{tabular}{lc}
\hline Major bleeding site & $\%(\mathrm{n})$ \\
\hline Gastrointestinal & $36(18)$ \\
Intramuscular/compartment syndrome & $24(12)$ \\
Intracranial & $16(8)$ \\
Urogenital & $12(6)$ \\
Retroperitoneal & $6(3)$ \\
Other* & $6(3)$ \\
ISTH major bleeding criteria & $6(3)$ \\
Fatal bleeding $\dagger$ & $46(23)$ \\
Critical area or organ $\ddagger$ & $48(24)$ \\
Blood transfusion $\S \pm$ hemoglobin fall $\mathbb{1}$ &
\end{tabular}

\footnotetext{
*Pericardial and subcutaneous/hematoma; ISTH, International Society on Thrombosis and Haemostasis

$\uparrow$ Fatal outcome within one week after major bleeding

$\ddagger$ Intracranial, retroperitoneal, pericardial and intramuscular with compartment syndrome

$\S$ Bleeding leading to transfusion of $\geq 2$ units of whole blood or red cells

II Bleeding causing a fall in hemoglobin level of $\geq 20 \mathrm{~g} / \mathrm{L}$
} 
Table 3 Risk of major bleeding (MB) by individual single nucleotide polymorphisms (SNPs) and number of prothrombotic risk alleles in patients with venous thromboembolism

\begin{tabular}{|c|c|c|c|c|}
\hline Risk allele & MB events $(n=50)$ & IR $(95 \% \text { CI })^{*}$ & $\mathrm{HR}(95 \% \mathrm{CI}) \dagger$ & $\mathrm{HR}(95 \% \mathrm{CI})+$ \\
\hline \multicolumn{5}{|l|}{ SNP (gene) } \\
\hline \multicolumn{5}{|l|}{ rs6025 $(F 5)$} \\
\hline 0 & 43 & $9.6(7.1-13.0)$ & Ref. & Ref. \\
\hline$\geq 1$ & 7 & $8.5(4.1-17.9)$ & $1.0(0.4-2.2)$ & $1.0(0.4-2.2)$ \\
\hline \multicolumn{5}{|l|}{ rs1799963 (F2) } \\
\hline 0 & 50 & $9.7(7.4-12.8)$ & Ref. & Ref. \\
\hline$\geq 1$ & 0 & - & - & - \\
\hline \multicolumn{5}{|l|}{ rs8176719 $(A B O)$} \\
\hline 0 & 17 & $10.2(6.3-16.4)$ & Ref. & Ref. \\
\hline$\geq 1$ & 33 & $9.1(6.5-12.8)$ & $1.0(0.5-1.7)$ & $1.0(0.5-1.7)$ \\
\hline \multicolumn{5}{|l|}{ rs2066865 (FGG) } \\
\hline 0 & 26 & $9.1(6.2-12.4)$ & Ref. & Ref. \\
\hline$\geq 1$ & 24 & $9.8(6.6-14.7)$ & $1.1(0.7-2.0)$ & $1.2(0.7-2.0)$ \\
\hline \multicolumn{5}{|l|}{ rs2036914 (F11) } \\
\hline 0 & 5 & $5.3(2.2-12.8)$ & Ref. & Ref. \\
\hline$\geq 1$ & 45 & $10.4(7.7-13.9)$ & $2.0(0.8-5.0)$ & $2.0(0.8-5.0)$ \\
\hline \multicolumn{5}{|l|}{ 5-SNP score } \\
\hline $0-1$ & 6 & $7.0(3.2-15.7)$ & Ref. & Ref. \\
\hline 2 & 15 & $11.3(6.8-18.7)$ & $1.7(0.7-4.5)$ & $1.7(0.7-4.5)$ \\
\hline 3 & 17 & $10.8(6.7-17.4)$ & $1.6(0.6-4.0)$ & $1.6(0.6-4.0)$ \\
\hline$\geq 4$ & 12 & $7.9(4.5-13.9)$ & $1.3(0.5-3.5)$ & $1.3(0.5-3.5)$ \\
\hline Per category increase & & & $1.0(0.8-1.3)$ & $1.0(0.8-1.3)$ \\
\hline
\end{tabular}

* IR, incidence rate per 100 person-years; HR, hazard ratio; CI, confidence interval

$\dagger$ Adjusted for age and sex

\$ Adjusted for age, sex and planned duration of anticoagulation 
Table 4 Risk of major bleeding (MB) by individual single nucleotide polymorphisms (SNPs) and number of prothrombotic risk alleles in patients with venous thromboembolism (VTE) without active cancer at the time of VTE diagnosis

\begin{tabular}{|c|c|c|c|c|}
\hline Risk allele & MB events $(\mathrm{n}=35)$ & IR $(95 \%$ CI $)$ & $\mathrm{HR}(95 \% \mathrm{CI}) \dagger$ & $\mathrm{HR}(95 \% \mathrm{CI})+$ \\
\hline \multicolumn{5}{|l|}{ SNP (gene) } \\
\hline \multicolumn{5}{|l|}{ rs6025 $(F 5)$} \\
\hline 0 & 28 & $7.5(5.2-10.9)$ & Ref. & Ref. \\
\hline$\geq 1$ & 7 & $9.5(4.5-19.9)$ & $1.5(0.7-3.5)$ & $1.6(0.7-3.6)$ \\
\hline \multicolumn{5}{|l|}{ rs1799963 (F2) } \\
\hline 0 & 35 & $8.1(5.8-11.3)$ & Ref. & Ref. \\
\hline$\geq 1$ & 0 & - & - & - \\
\hline \multicolumn{5}{|l|}{ rs8176719 $(A B O)$} \\
\hline 0 & 13 & $9.6(5.5-16.5)$ & Ref. & Ref. \\
\hline$\geq 1$ & 22 & $7.1(4.7-10.6)$ & $0.8(0.4-1.5)$ & $0.8(0.4-1.5)$ \\
\hline \multicolumn{5}{|l|}{ rs2066865 (FGG) } \\
\hline 0 & 17 & $7.1(4.4-11.3)$ & Ref. & Ref. \\
\hline$\geq 1$ & 18 & $8.8(5.5-13.9)$ & $1.3(0.7-2.5)$ & $1.4(0.7-2.6)$ \\
\hline \multicolumn{5}{|l|}{ rs2036914 (F11) } \\
\hline 0 & 2 & $2.6(0.6-10.4)$ & Ref. & Ref. \\
\hline$\geq 1$ & 33 & $8.9(6.4-12.6)$ & $3.6(0.9-15.0)$ & $3.5(0.8-14.7)$ \\
\hline \multicolumn{5}{|l|}{ 5-SNP score } \\
\hline $0-1$ & 4 & $5.8(2.2-15.5)$ & Ref. & Ref. \\
\hline 2 & 8 & $7.2(3.6-14.4)$ & $1.4(0.4-4.8)$ & $1.4(0.4-4.7)$ \\
\hline 3 & 14 & $10.9(6.5-18.4)$ & $2.0(0.6-6.0)$ & $2.0(0.7-6.2)$ \\
\hline$\geq 4$ & 9 & $6.5(3.4-12.5)$ & $1.3(0.4-4.3)$ & $1.3(0.4-4.4)$ \\
\hline Per category increase & & & $1.1(0.8-1.5)$ & $1.1(0.8-1.5)$ \\
\hline
\end{tabular}

* IR, incidence rate per 100 person-years; HR, hazard ratio; CI, confidence interval $\dagger$ Adjusted for age and sex

$\$$ Adjusted for age, sex and planned duration of anticoagulation 
Table 5 Risk of major bleeding (MB) by number of prothrombotic risk alleles in subgroups of venous thromboembolism

\begin{tabular}{|c|c|c|c|c|}
\hline DVT $(n=387)$ & MB events & IR $(95 \%$ CI $)$ & $\mathrm{HR}(95 \% \mathrm{CI}) \dagger$ & $\mathrm{HR}(95 \% \mathrm{CI})$ \\
\hline \multicolumn{5}{|l|}{ 5-SNP score } \\
\hline $0-1$ & 3 & $6.2(2.0-19.2)$ & Ref. & Ref. \\
\hline 2 & 10 & $13.9(7.5-25.8)$ & $2.2(0.6-8.0)$ & $2.2(0.6-8.1)$ \\
\hline 3 & 10 & $10.7(5.7-19.8)$ & $1.7(0.5-6.2)$ & $1.7(0.5-6.2)$ \\
\hline$\geq 4$ & 5 & $5.7(2.4-13.1)$ & $1.0(0.2-4.0)$ & $1.0(0.2-4.0)$ \\
\hline Per category increase & & & $0.9(0.6-1.3)$ & $0.9(0.6-1.3)$ \\
\hline \multicolumn{5}{|l|}{$\mathrm{PE}(\mathrm{n}=289)$} \\
\hline \multicolumn{5}{|l|}{ 5-SNP score } \\
\hline $0-1$ & 3 & $8.2(2.6-25.4)$ & Ref. & Ref. \\
\hline 2 & 5 & $8.2(3.4-19.6)$ & $1.2(0.3-5.3)$ & $1.3(0.3-5.5)$ \\
\hline 3 & 7 & $11.0(5.3-23.1)$ & $1.5(0.4-5.7)$ & $1.6(0.4-6.1)$ \\
\hline$\geq 4$ & 7 & $10.9(5.2-22.9)$ & $1.8(0.5-7.0)$ & $1.9(0.5-7.6)$ \\
\hline Per category increase & & & $1.2(0.8-1.8)$ & $1.2(0.8-1.9)$ \\
\hline \multicolumn{5}{|l|}{ Provoked $(\mathrm{n}=392)$} \\
\hline \multicolumn{5}{|l|}{ 5-SNP score } \\
\hline $0-1$ & 4 & $8.2(3.1-21.7)$ & Ref. & Ref. \\
\hline 2 & 11 & $14.6(8.1-26.4)$ & $2.0(0.6-6.3)$ & $2.0(0.6-6.5)$ \\
\hline 3 & 12 & $15.6(8.9-27.5)$ & $1.9(0.6-6.0)$ & $2.0(0.6-6.2)$ \\
\hline$\geq 4$ & 8 & $11.5(5.7-23.0)$ & $1.6(0.5-5.4)$ & $1.7(0.5-5.6)$ \\
\hline Per category increase & & & $1.1(0.8-1.5)$ & $1.1(0.8-1.5)$ \\
\hline \multicolumn{5}{|l|}{ Unprovoked $(n=284)$} \\
\hline \multicolumn{5}{|l|}{ 5-SNP score } \\
\hline $0-1$ & 2 & $5.5(1.4-22.1)$ & Ref. & Ref. \\
\hline 2 & 4 & $6.9(2.6-18.3)$ & $1.4(0.3-7.9)$ & $1.4(0.3-7.9)$ \\
\hline 3 & 5 & $6.2(2.6-14.9)$ & $1.2(0.2-6.3)$ & $1.2(0.2-6.3)$ \\
\hline$\geq 4$ & 4 & $4.8(1.8-12.9)$ & $1.0(0.2-5.6)$ & $1.0(0.2-5.6)$ \\
\hline Per category increase & & & $1.0(0.6-1.6)$ & $1.0(0.6-1.6)$ \\
\hline
\end{tabular}

SNP, single nucleotide polymorphism; DVT, deep vein thrombosis; PE, pulmonary embolism

* IR, incidence rate per 100 person-years; HR, hazard ratio; CI, confidence interval

$\dagger$ Adjusted for age and sex

$\$$ Adjusted for age, sex and planned duration of anticoagulation 


\section{Legends to figures}

Figure 1. Distribution (\%) of patients with incident venous thromboembolism (VTE) across number of risk alleles of the 5-single nucleotide polymorphism (SNP) score.

Figure 2. Cumulative incidences (1-Kaplan-Meier) of major bleeding according to categories of risk alleles of the 5-single nucleotide polymorphism (SNP) score in overall venous thromboembolism (VTE) (A) and after excluding subjects with active cancer at the time of VTE diagnosis (B). 
Figure 1

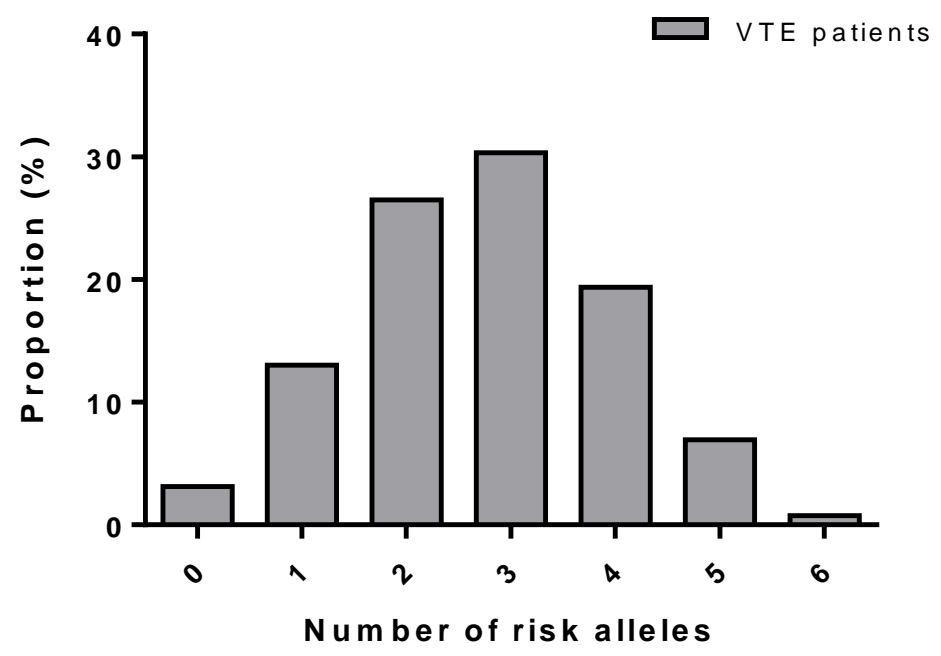


Figure 2A

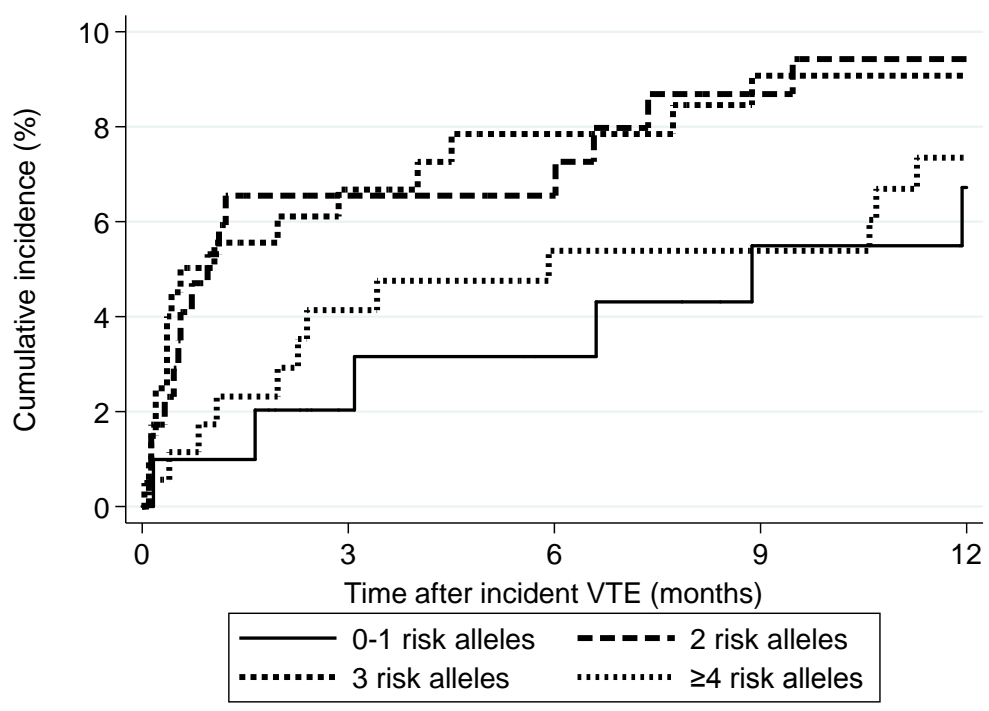

Figure 2B

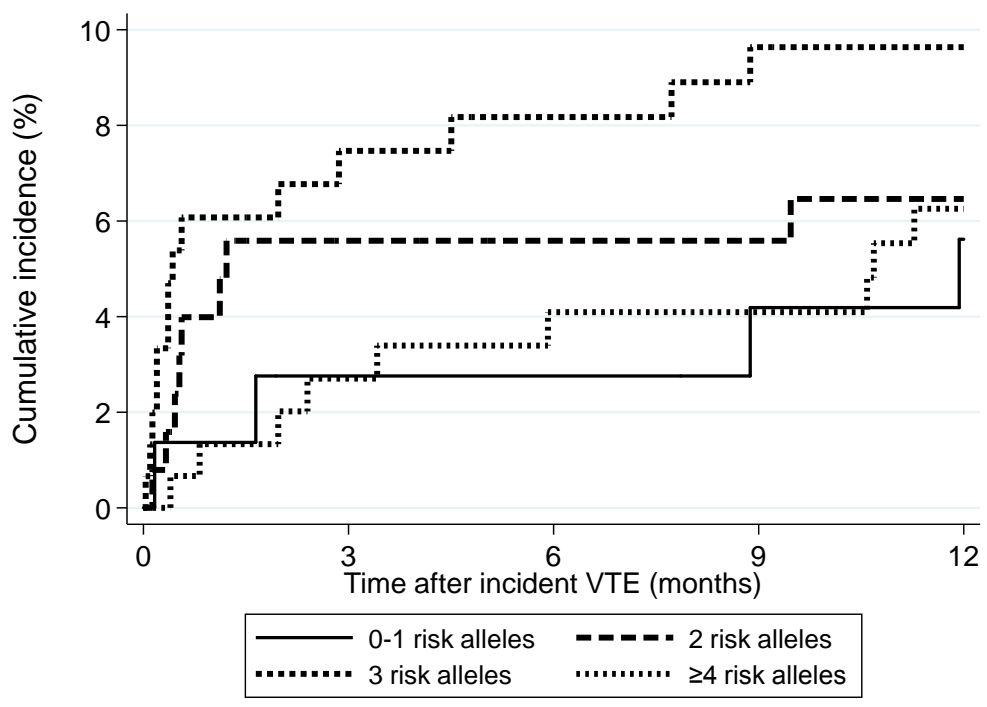




\section{Supplementary material}

Supplementary Table 1 Risk of major bleeding (MB) by individual single nucleotide polymorphisms (SNPs) and number of prothrombotic risk alleles during the first three months after venous thromboembolism

\begin{tabular}{|c|c|c|c|}
\hline Risk allele & MB events $(n=33)$ & IR $(95 \% \mathrm{CI})$ & $\mathrm{HR}(95 \% \mathrm{CI}) \dagger$ \\
\hline \multicolumn{4}{|l|}{ SNP (gene) } \\
\hline \multicolumn{4}{|l|}{ rs6025 (F5) } \\
\hline 0 & 29 & $23.8(16.6-34.3)$ & Ref. \\
\hline$\geq 1$ & 4 & $18.0(6.8-47.9)$ & $0.8(0.3-2.4)$ \\
\hline \multicolumn{4}{|l|}{ rs1799963 (F2) } \\
\hline 0 & 33 & $23.5(16.7-33.0)$ & Ref. \\
\hline$\geq 1$ & 0 & - & - \\
\hline \multicolumn{4}{|l|}{ rs8176719 $(A B O)$} \\
\hline 0 & 9 & $19.7(10.3-37.9)$ & Ref. \\
\hline$\geq 1$ & 24 & $24.4(16.4-36.4)$ & $1.3(0.6-2.8)$ \\
\hline \multicolumn{4}{|l|}{ rs2066865 $(F G G)$} \\
\hline 0 & 15 & $19.3(11.6-32.0)$ & Ref. \\
\hline$\geq 1$ & 18 & $27.2(17.1-43.2)$ & $1.5(0.7-3.0)$ \\
\hline \multicolumn{4}{|l|}{ rs2036914 (F11) } \\
\hline 0 & 3 & $11.7(3.8-36.4)$ & Ref. \\
\hline$\geq 1$ & 30 & $25.3(17.7-36.2)$ & $2.2(0.7-7.0)$ \\
\hline \multicolumn{4}{|l|}{ 5-SNP score } \\
\hline $0-1$ & 2 & $8.2(2.2-34.6)$ & Ref. \\
\hline 2 & 11 & $29.5(16.5-53.7)$ & $3.8(0.8-17.2)$ \\
\hline 3 & 13 & $29.9(17.4-51.6)$ & $3.5(0.8-15.7)$ \\
\hline$\geq 4$ & 7 & $17.3(8.2-36.3)$ & $2.2(0.5-10.7)$ \\
\hline Per category increase & & & $1.1(0.8-1.5)$ \\
\hline
\end{tabular}

